\title{
Concentric Microwaves of Henle's Fiber Layer: in Association with Cystoid Macular Edema*
}

\author{
J. Reimer Wolter \\ The Departments of Ophthalmology and Pathology of the University of Michigan Hospitals, Ann Arbor, Michigan, 48109, USA
}

\begin{abstract}
Fully developed microwaves of Henle's fiber layer all around a foveola exhibiting cystoid changes and localized detachment are demonstrated in an eye, that was proptosed and exposed due to advanced basal cell carcinoma. The significance of microwaves in Henle's fiber layer among other reactive changes of the foveal retina is discussed.
\end{abstract}

Zusammenfassung. Voll entwickelte Mikrowellen in Henle's Faserschicht rund um eine Foveola herum, die auch zystoide Veränderungen und lokale Netzhautablösung zeigt, werden in einem Auge dargestellt, das wegen Basalzellcarcinom Proptosis und mangelnden Lidschutz aufwies. Die Bedeutung von Mikrowellen in Henle's Faserschicht im Verhältnis zu anderen reaktiven Veränderungen in der fovealen Netzhaut wird erörtert.

Concentric microwaves have been studied in their histological nature in a case of orbital tumor pressure on the globe from behind [1]. It has been concluded, that the concentric folds under those conditions probably represent a protective adjustment of the central retina to minimize distortion of the foveolar cones. To give a histological description of concentric microwaves in association with cystoid macular edema (CME) is the purpose of the present paper.

The microarchitecture of the retinal foveola and its adjustments under pathological conditions are very important for the preservation of the proper alignment of the foveolar cones. These are the cones, that are responsible for central vision, of course. Under normal conditions, the foveola is a valley. The foveolar cones at the bottom of this valley extend through the openings in the outer limiting membrane towards the pigment epithelium. Their orderly arrangement is protected by a rather stable proteinaceous intercellular substance only. In the space between outer limiting membrane of the retina and the pigment epithelium they have no glial support. Disorder of foveolar cones in a late stage of CME has recently been demonstrated in a histopathological study [2]. The cell bodies with the nuclei of the cone cells are arranged along the anterior surface of the outer limiting membrane. The synapses between the cone cells and the cells of the inner nuclear layer take place next to the inner nuclear layer and these are represented in histolog-

\footnotetext{
* Supported by The Research To Prevent Blindness, Inc., New York, N.Y., USA
}

ical cross sections of the retina by a visible line sometimes known as middle limiting membrane. In and around the foveola the neuronal connection between cone cells and bipolar cells in the inner nuclear layer is shifted to the periphery and the neuronal processes of the cone cells are extended. The resulting star burst of neuronal fibers and associated glial processes is known as Henle's fiber layer. Normally, this is laying flat on the outer nuclear layer and all its fibers are firmly packed, straight and of regular arrangement (Fig. 1) [3].

Macular edema or early CME typically go along with accumulation of an extracellular transudate in Henle's fiber layer $[2,49]$. This probably is a reversible change and Henle's fiber layer greatly thickens in the process of fluid accumulation. Henle's fibers "stand up" from the flat position, that is normal. With persisting edema microcystoid and cystoid changes develop - not only in Henle's fiber layer, but also in the inner and outer nuclear layers of the foveolar zone [4]. With time of persisting fluid leakage, the transudate tends to change into a more permanent and more proteinaceous exudate of loose fibrinous, sero-fibrinous or even serous nature. The difference between this transudate or exudate and a so-called deep hard exudate is, that the fluids in the spaces of CME typically contain no macrophages - or at least only very few [4]. Deep hard exudates, in contrast, exhibit numerous lipid filled cells [10]. Degeneration of neurons and glial structures goes along with progressive swelling, exudate and cystoid changes in the foveol retina. The normal valley of the foveola flattens out in the process and with enlarging cystoid spaces, finally, a mound develops in its place [4]. The formation of this mound is enhanced by localized detachment of the foveolar cones and/or vertical folding of the outer retinal layers, that is commonly associated with CME $[2,4-6,8,9]$.

With the aim to gain insight into the detailed tissue processes, that take place in early CME, a case is presented, that allows for a good demonstration of the involvement of Henle's fiber layer in an eye with combined proptosis and CME.

\section{Case Report}

The eye of this 57 year-old female was already used once for the demonstration of pathological details in cystoid macular edema [4]. The findings in this eye are of special value, because the eye was not enucleated. It was removed along 


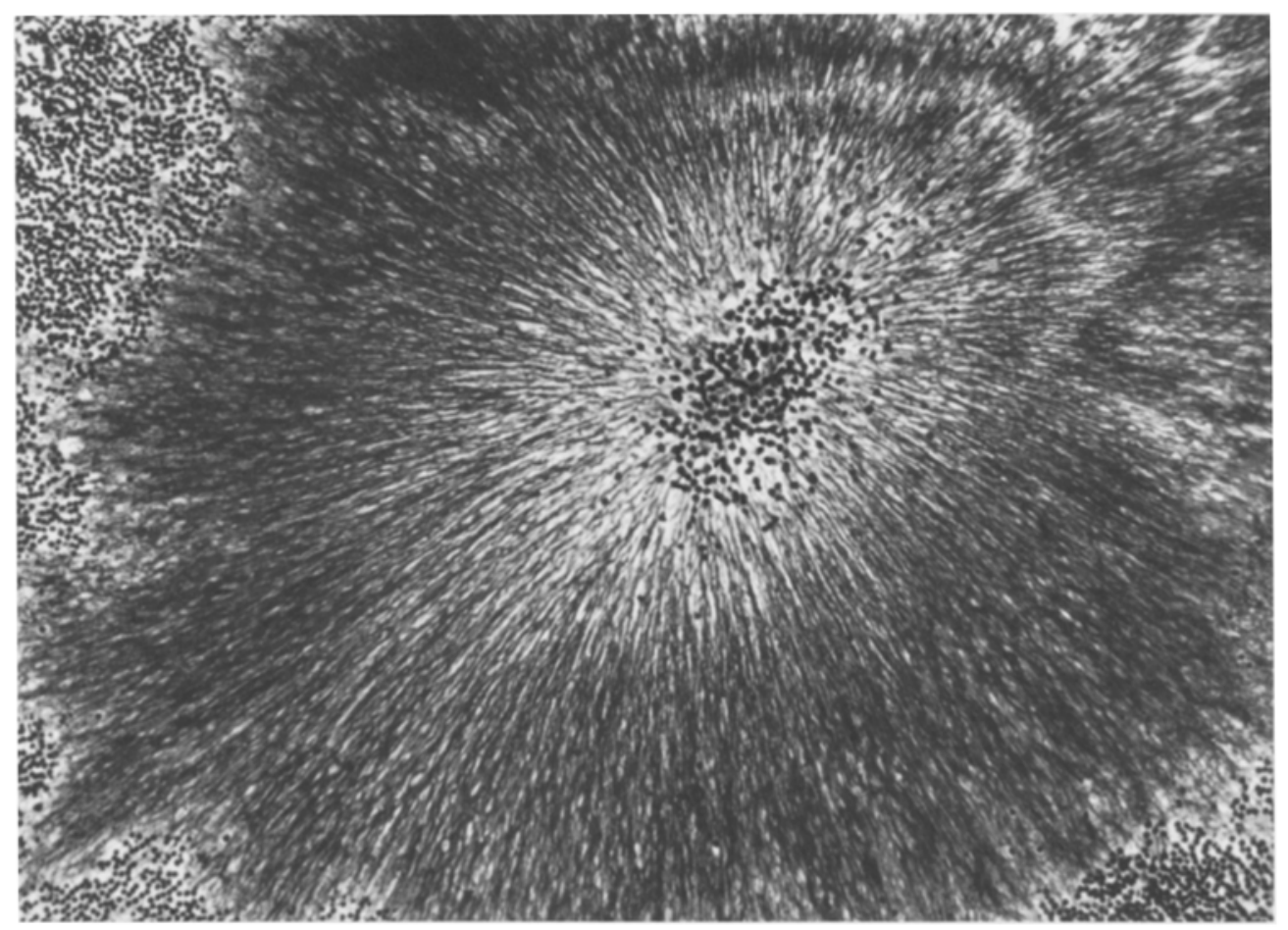

Fig. 1. (Wolter) Flat section through Henle's fiber layer of the normal human retina. The top of the central accumulation of cone nuclei is seen in the center. The star burst of radiating fibers are Henle's fibers in their normal arrangement. Nuclei of the inner nuclear layer are seen in peripheral corners of the photograph. - Flat frozen section, silver stain, photomicrograph $\times 150$

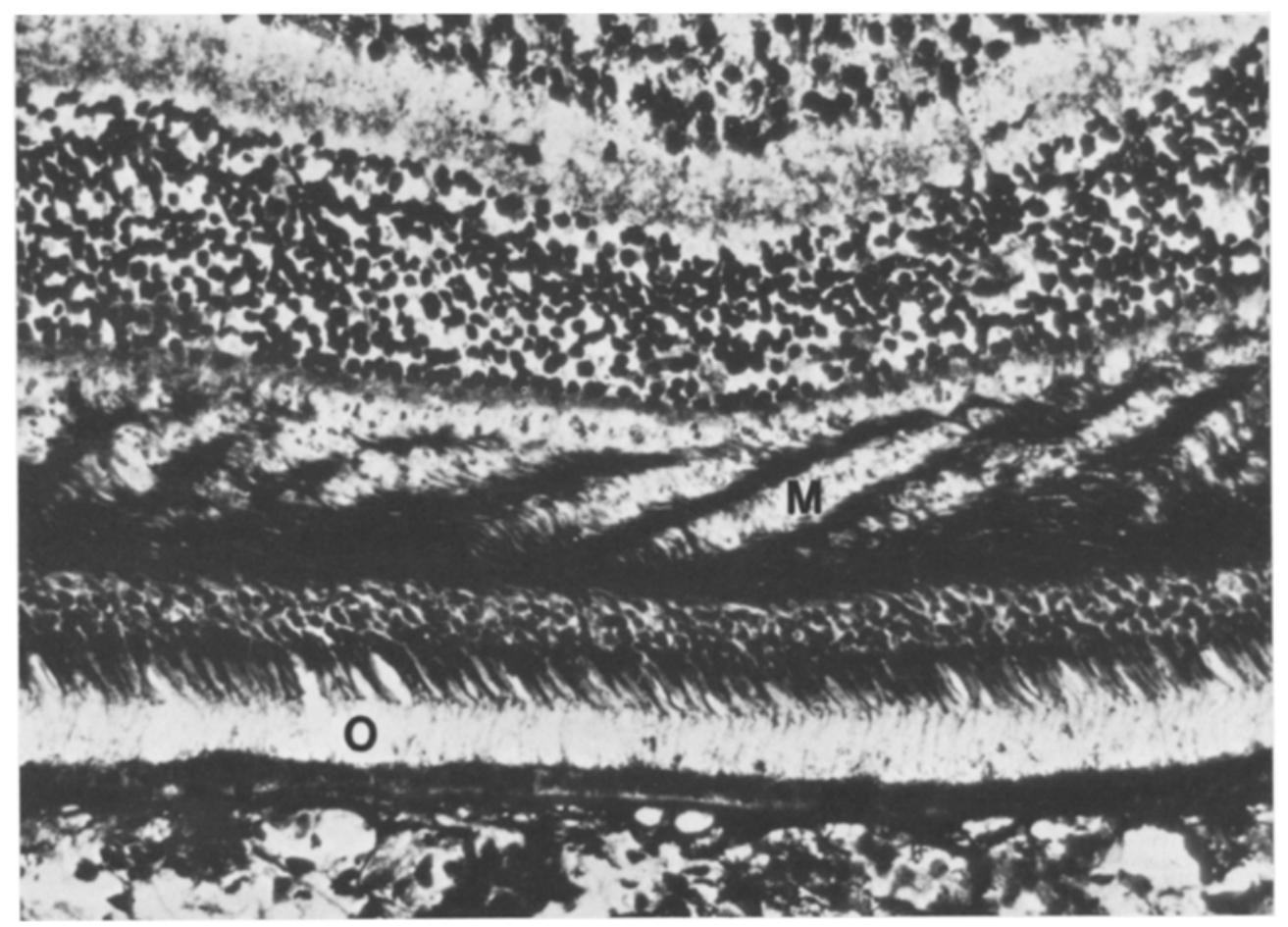

Fig. 2. (Wolter) Horizontal cross section through the foveola of the present eye exhibiting cystoid changes $(C)$ in the center of Henle's fiber layer, detachment of central cones $(D)$ and microwaves of the parafoveolar Henle's fibers $(M)$. The foveola has no inner limiting membrane; the end of this membrane $(L)$ is seen on the right. The choroid with its vessels $(V)$ and with the pigment epithelium on top is seen below in the photograph. - Frozen section, silver stain, photomicrograph $\times 150$ 


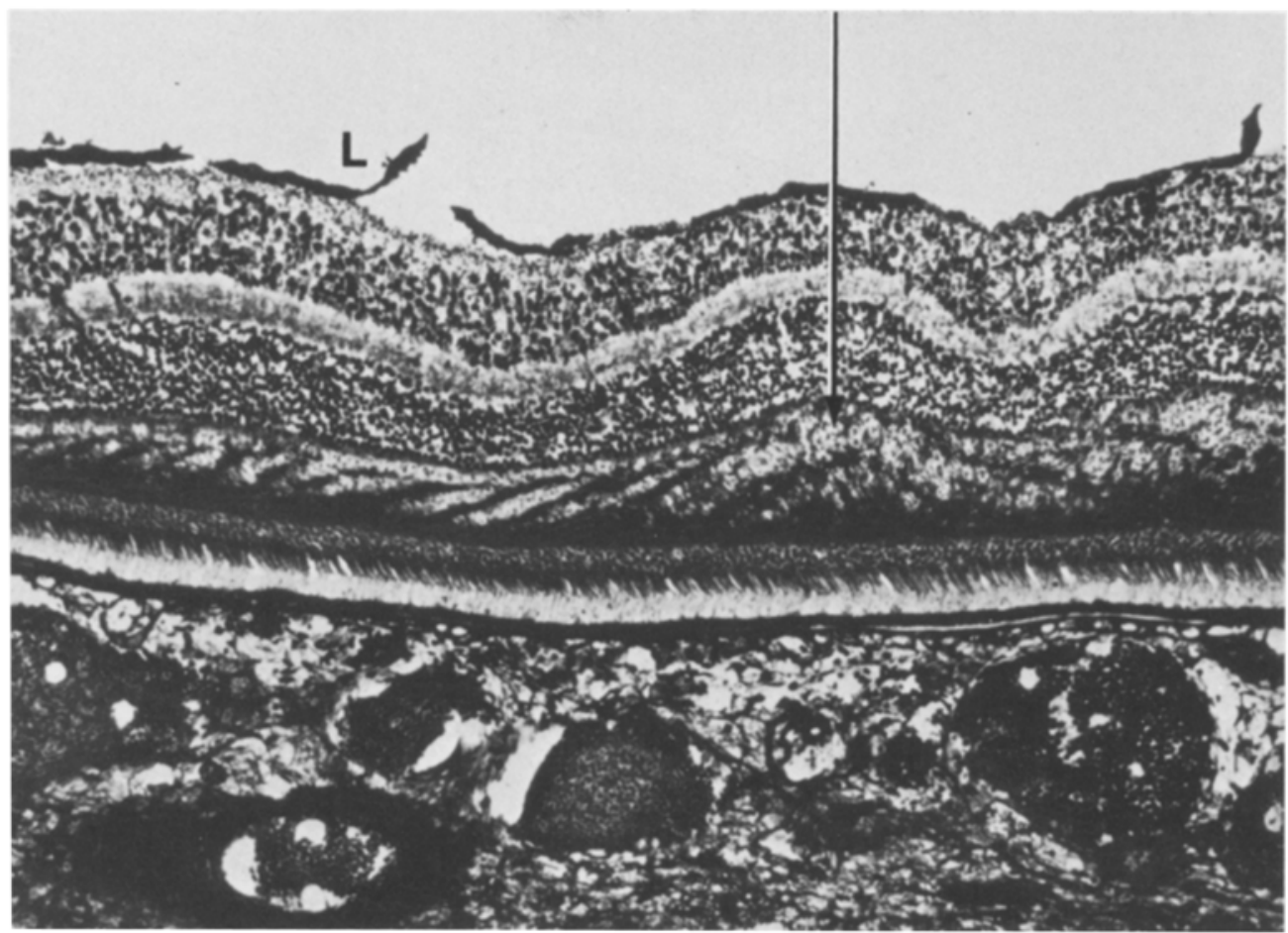

Fig. 3. (Wolter) Horizontal cross section of foveal retina in a plane slightly below the foveola. The microwaves in Henle's layer are centered on the foveola (arrow). Slight folding involves the inner retinal layers with the inner limiting membrane $(L)$ artificially broken in a few areas. At this parafoveal plane there is no detachment of central cones. The choroid is seen in the lower part of the photograph. - Frozen section, silver stain, photomicrograph $\times 100$

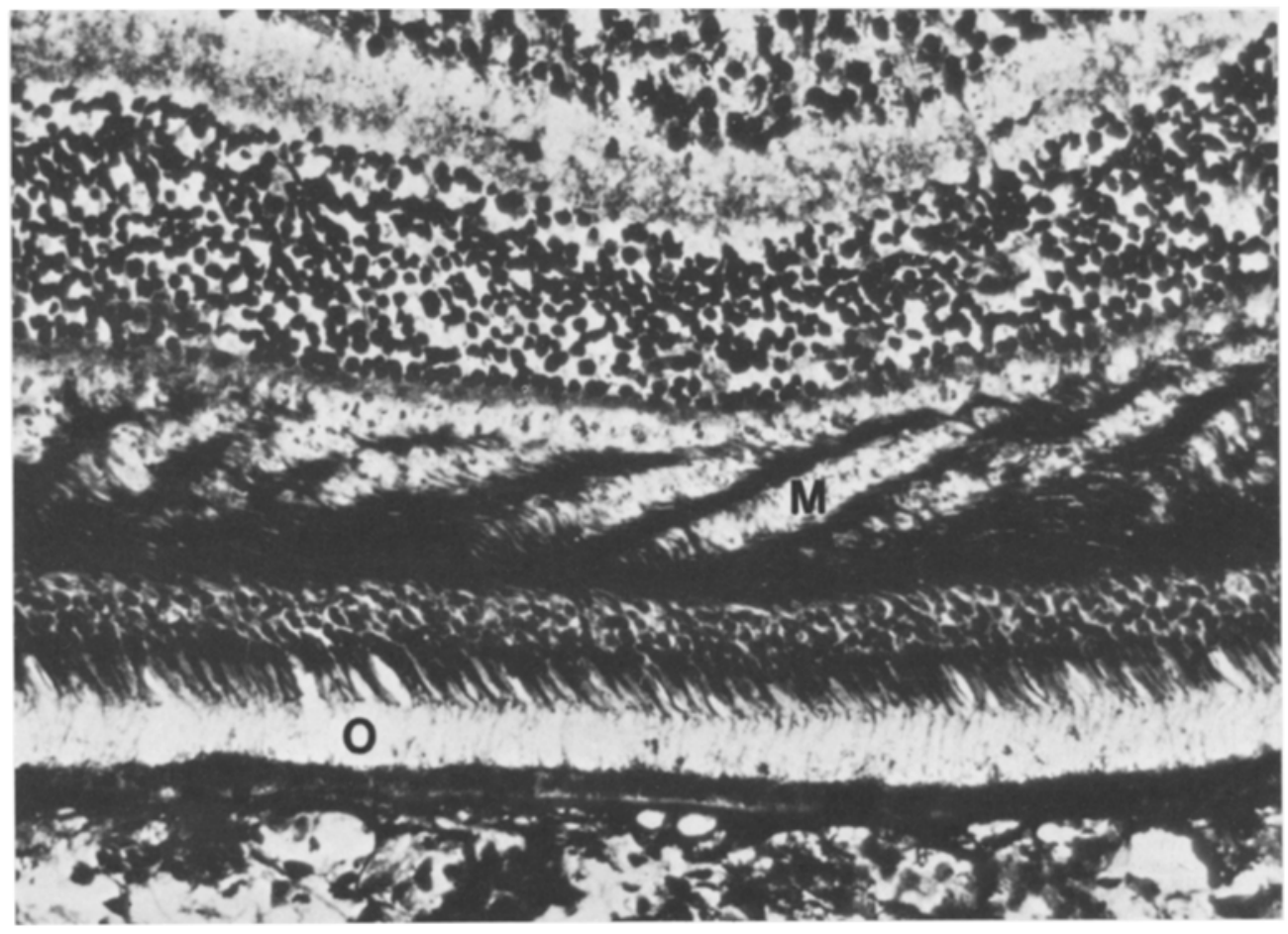

Fig. 4. (Wolter) Higher power of one part of the view given in Fig. 3 to demonstrate the regular nature of the microwaves ( $M$ ) in Henle's fiber layer. The cones with their outer processes $(0)$, the pigment epithelium and the choriocapillaris are seen below. - Frozen section, silver stain, photomicrograph $\times 200$ 


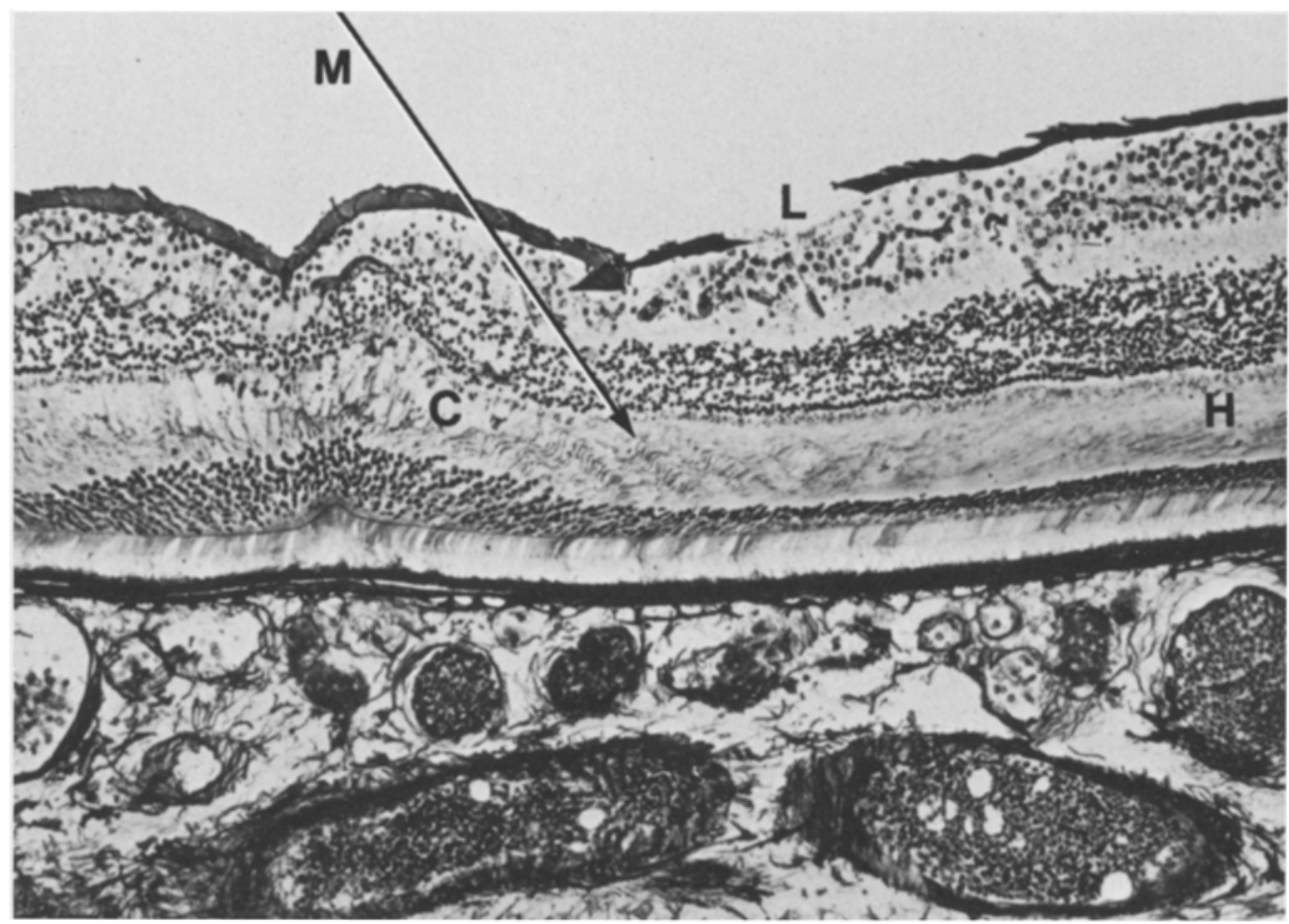

Fig. 5. (Wolter) Horizontal cross section of the foveal retina slightly above the foveola after a light silver stain clearly showing the microwaves $(M)$ in the swollen Henle's fiber layer $(H)$. The center of Henle's fiber layer has early cystoid changes $(C)$. The foveola exhibits a sharp vertical fold, that is associated with detachment of the foveola seen in Fig. 2. The inner limiting membrane is folded slightly along with the inner retinal layers and it shows an artificial defect $(L)$. Frozen section, silver stain, photomicrograph $\times 100$

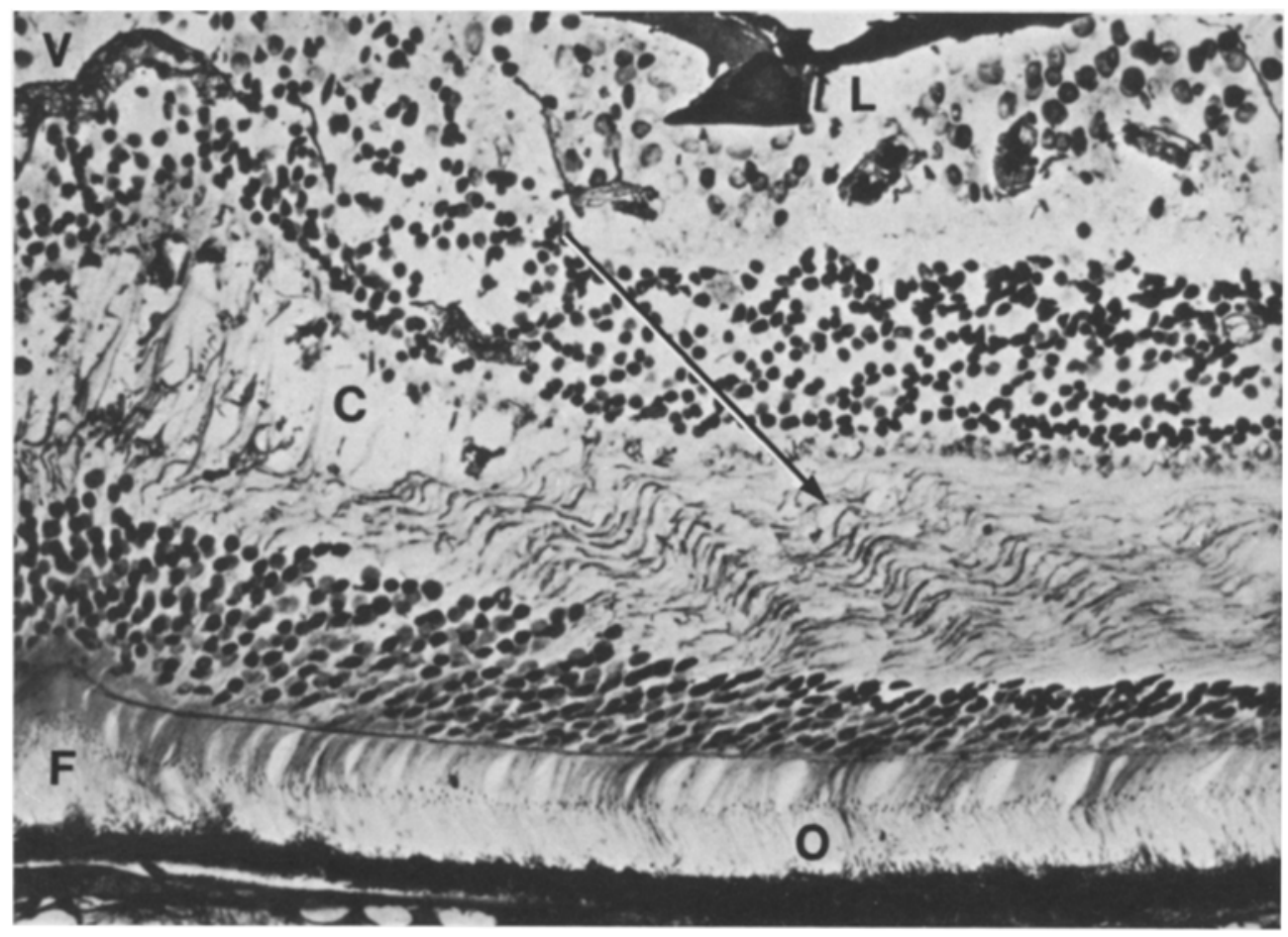

Fig. 6. (Wolter) Higher power of a part of the view given in Fig. 5 to show the lightly stained microwaves (arrow) and the early cystoid changes $(C)$ in detail. The outer limiting membrane has a fold $(F)$ and the central cones are slightly detached in this plane. All around the fold the outer cone segments $(O)$ are in place and in contact with the pigment epithelium. - Frozen section, silver stain, photomicrograph $\times 200$ 
with a good part of the patient's face including the orbit and the surrounding orbital bones because of infiltrating basal cell carcinoma. This piece of face was fixed in Formalin as a whole and the eye was removed from the orbit after fixation. The eye was proptosed and vision was very poor due to corneal exposure and scarring in virtual absence of eyelids. The eye was of normal size and grossly exhibited an early cataract and papilledema in addition to the corneal scarring, when it was opened in a horizontal plane. The posterior pole with fovea and optic nerve head was isolated, cut in frozen sections and stained with several modifications of the silver method of Del Rio Hortega for the demonstration of neurons.

The papilledema, central cystoid degeneration in Henle's fiber layer and slight detachment with vertical folding of the outer retinal layers found in this eye were demonstrated and discussed in the earlier publication [4] (Figs. 1, 2 and 3). Figure 2 of the present paper serves for the orientation of the reader; it shows the parafoveal areas of microwaving in the swollen Henle's fiber layer on both sides of the central cystoid changes in a horizontal cross section. These cystoid changes together with the elevation of the outer retinal layers result in a distinct mound, that replaces the normal valley of the foveola.

Figure 3 shows a horizontal cross section slightly inferior to the foveola of the same eye. All retinal layers are represented. Henle's fiber layer is swollen and the inferior extension of one of the petaloid folds of the inner retinal layers extending from the foveola in an inferior direction is visible in this plane. There is no detachment of outer limiting membrane and parafoveolar cones. The regular waves of Henle's fibers arranged around the foveola are clearly visible in their concentric arrangement around the foveola. Figure 4 shows a higher power view of these regular waves nasal and inferior to the foveola. Figures 4 and 5 are views of a less intense silver stain of another parafoveolar section cut superior to the foveola. The dip of the inner retinal layers seen in the center of this section is not the foveola, but it represents the valley of one of the petaloid folds of the inner retinal layers found in this case. In Fig. 5 Henle's fiber layer shows a portion of the central cystoid degeneration. Nasal to the cystoid changes there are the beautiful microwaves in their regular arrangement - more impressive due to the fact, that the central processes of the cone cells (Henle's fibers) are only slightly stained (Fig. 6). The outer retina in Figs. 5 and 6 exhibits the foldlike elevation of foveolar cones and outer limiting membrane, that has been demonstrated and discussed extensively in earlier studies on CME [2, 4-6].

\section{Discussion}

Concentric microwaves in Henle's fiber layer have been shown to occur in cases of proptosis ${ }^{1}$ and also in cases of advanced CME $[4,5,8]$. The microwaves in CME are not usually as complete and well developed, as they are in cases with tumor pressure on the posterior sclera. The eye in the present case was involved with both, proptosis due to tumor pressure on the globe from behind and CME probably resulting from uveitis secondary to exposure keratitis. The occurrence of waves in Henle's fiber layer indicates, that the fibers in this layer are not very elastic. The regular arrangement of the microwaves reveals a tendency for very orderly reactions in this layer. Microwaves in cases with tumor pressure on the posterior sclera must be the result of shortening of the posterior ocular wall resulting from this pressure. Microwaves in CME probably result from expansion of the foveolar retina due to cystoid changes - often in combination with additional foveolar detachment. It is no surprise, that the combination of posterior pressure on the eye with papilledema and mound-like CME with limited foveolar detachment in the present case has resulted in the most regular and beautiful microwaves all around the foveola. In general, these waves are best seen in flat sections through the central retina, but in the present case their regular and concentric arrangement is very well visible in cross sections. It is important to emphasize, that microwaves only seem to occur in cases with a swollen central retina exhibiting fluid accumulation in Henle's fiber layer.

Progressive CME leads to destruction with cystoid changes in Henle's fiber layer. The phase with presence of microwaves, thus, is only short in serious cases. However, the advanced stages of CME often still exhibit segmental patches of these microwaves, which probably are left from an earlier stage with more regular swelling and less destruction. Pull on the central retina due to membranes or tumors is an additional cause of microwaves in Henle's fiber layer. The folds are typically incomplete and excentric under these more complicated conditions.

The occurrence of microwaves in macular edema and/or $\mathrm{CME}$ is one of the slight adjustment possibilities, that nature has to protect an important function. The waves are partly due to fluid accumulation in the central retina and I believe, that they may be a reversible change. The microwaves, thus, indicate equal distribution of fluid all through the foveolar structures to avoid as long as possible pull on the foveolar cones, that causes disorder and distortion resulting in difficulties with central vision.

\section{References}

1. Wolter JR (1981) Concentric "Microwaves" of Henle's Fiber Layer: Associated with Horizontal Folding, v Graefes f Ophth 216:31-39

2. Wolter JR (1981) Foveal Pathology Following Anterior Segment Injury, Ophth Surg 12:661-665

3. Polyak S (1957) The Vertebrate Visual System. The University of Chicago Press

4. Wolter JR (1981) The Histopathology of Cystoid Macular Edema, v Graefes Arch f Ophth 216:85-101

5. Wolter JR, Croasdale RE, Bahn CF (1980) Reactions to an Anterior Chamber Lens - Two Years After Implantation. Ophth Surg 11:794-800

6. Wolter JR (1981) Vertical Folds of Central retina and Choroid in Sudden Ocular Decompression, Ophth Surg 12:190-194

7. Wolter JR (1981) Cystoid Macular Edema in Vitreo-Retinal Traction, Ophth Surg 12:900-904

8. Wolter JR Three Basic Types of Foveal Involvement in Choroidal Melanomas, in print v Graefe's Arch $f$ Ophth

9. Wolter JR (1982) Foveal Pathology in Ciliary Body Melanoma Ophth Surg 13:309-311

10. Wolter JR, Goldsmith RI, Phillips RL (1957) Histopathology of the Star Figure of the Macular Area in Diabetic and Angiospastic Retinopathy, AMA Arch Ophth 57:376-385

Received March 29, 1982 